La primera obra rusa traducida al español fue la Oda al Ser NOTA · 103-112

Supremo, una joya de la obra de Gavrila Romanovich Derzhavin, considerado como el mejor poeta ruso del siglo Xvill y el primero entre los poetas y escritores de Rusia en adquirir fama internacional. Este hecho es cierto, refrendado por muchas investigaciones realizadas acerca de las traducciones de la literatura rusa. El trabajo tiene como objetivo establecer la fecha y el autor al que se debe la primera traducción de la oda de Derzhavin, ya que siempre se ha creido que era una traducción de un autor anónimo. Se describen y se comparan las opiniones de diferentes investigadores de este tema y se lleva a cabo un análisis de las dos traducciones al español más antiguas publicadas en las revistas españolas. Como resultado de este trabajo se logra identificar al autor de la primera traducción de la Oda al Ser Supremo al español, establecer la fecha del primer artículo sobre la literatura rusa publicado en España, así como concretar el nombre de la revista en la que aparece.

PALABRAS CLAVE: traducción, primeras relaciones literarias entre España y Rusia, la oda Dios de Derzhavin, Alberto Lista

\title{
Análisis y estudio de la primera traducción de la Oda al Ser Supremo de Derzhavin al español
}

\section{Analysis and study on the first Spanish translation of the Ode to God by Derzhavin}

The first Russian work translated into Spanish was the "Ode to God", a masterpiece by Gavrila Romanovich Derzhavin, the best Russian poet of the XVIII century and the first among the Russian writers to acquire international fame. This fact is considered indisputable and is endorsed by studies on translations of the Russian literature. The aim of the present article is to establish the date of the first translation of the Ode and the name of its author since till now the translator was considered as anonymous. We discuss and compare arguments of different researches of this subject and carry out an analysis of the first two Spanish translations of this poem. As a result of this study the name of the author of the first translation of the "Ode to God" into Spanish is discovered, as well as the publishing date of the first article about Russian literature in Spain and the name of the journal in which it appeared.

KEY WORDS: translation, the first relations between Spanish and Russian literature, ode God by Derzhavin, Alberto Lista 


\section{INTRODUCCIÓN}

La Oda al Ser Supremo, ${ }^{\mathrm{I}}$ escrita en I784 por Gavrila Románovich Derzhavin, ${ }^{2}$ el poeta más

104 importante del clasicismo ruso, fue la obra más famosa de la literatura rusa del siglo xviı no solo en Rusia, sino también en muchos países europeos, asimismo en Japón. Según el biógrafo de Derzhavin, Jacob Grot³ (I864:I9o), esta oda adquirió casi inmediatamente fama europea gracias a sus traducciones al alemán, francés, inglés, italiano, español, checo, latín y japonés. 200 años más tarde George Schanzer (I968:8I9), hispanista americano, especialista en el tema de las relaciones interculturales entre Rusia y España, artífice de la primera bibliografía de las obras rusas traducidas al castellano, afirmó que Derzhavin era el mejor poeta ruso del siglo XVIII y el primero que gozó de fama internacional. He aquí una breve información acerca de la amplia popularidad de la poesía de Derzhavin y, particularmente, de su Oda al Ser Supremo.

Gavrila Románovich Derzhavin (I743-18r6) fue el máximo representante de la época de la Ilustración y del clasicismo ruso, pero también fue la figura pionera en la poesía rusa, poetainnovador que experimentó con diferentes tipos de ritmos y rimas, sonidos e imágenes, combinando el léxico de estilos lingüísticos opuestos. Todo esto le convirtió en el gran maestro de la literatura rusa antes de la época de Alexander Pushkin. Empezó a publicar sus odas en I776, pero su fama literaria comenzó en 1783 con la

I Con éste título la oda Dios (en ruso: Bog) de Derzhavin fue publicada en el siglo xix en las revistas de España y América Latina.

2 Para la transliteración de las palabras rusas seguimos el sistema del Servicio de Traducción Española del Parlamento Europeo (2005) [http://es.wikipedia.org/wiki/ Romanizaci\% $\mathrm{C}_{3} \% \mathrm{~B}_{3}$ n_del_ruso].

3 Jacob Karlovich Grot (1812-I893), filólogo y académico ruso, publicó en los años I864-I883 en St. Petersburgo la edición académica de todas las obras de Derzhavin en 9 volúmenes con una biografía del poeta y comentarios. oda Felitsa, dedicada a Catalina la Grande, la «filósofo en trono». ${ }^{4}$ Como se sabe, la emperatriz rusa estaba orgullosa por su correspondencia amistosa con filósofos tan representativos de la época de la Ilustración francesa como Voltaire, Diderot y D'Alembert y por eso la llamaban así. Durante el reinado de Catalina II, los literatos rusos estudiaron las influencias clásicas y europeas que inspiraron la Ilustración rusa.

Gavrila Derzhavin, nacido en Kazán, muy lejos de San Petersburgo, la capital del imperio ruso, empezó su carrera como un soldado y al final llegó a ocupar el puesto de secretario de la emperatriz Catalina la Grande. Los temas principales de su poesía fueron temas filosóficoreligiosos e históricos. Derzhavin compuso varios poemas morales, satíricos, anacreónticos y religiosos, entre los cuales destaca su poema filosófico Oda al Ser Supremo, en el que le preocupa la relación entre el hombre y Dios en la época de Ilustración. Derzhavin niega el ateísmo de los filósofos franceses y expresa su admiración ante el mundo, ante el Cosmos, y más que nada ante el ser humano, gracias a quien se une materia y alma:

¡Soy rey, esclavo, soy insecto y Dios! [La traducción es nuestra].

(Я изарь - я рабъ, 一я червь -я Богъ!) (Grot, I864: I99).

Ya tsar — ya rab, —ya cherv', —ya Bog!).

Gracias al ser humano, según el poeta, existe Dios:

4 La definición de Catalina la Grande como «filósofo en trono» pertenece a los políticos extranjeros. Entre los últimos libros sobre el tema hay que mencionar el libro del profesor de Estudios Eslavos de la Universidad Católica de Lovaina Emmanuel Waegemans (20IO) con el mismo título: De filosofe op de troon. Het literaire werk van Catherina II van Rusland. Antwerpen: Benerus.

5 Para el presente trabajo nos hemos servido de la edición de las obras de Derzhavin de Grot (1864) con ortografía del siglo XIX. 
¡Yo soy! ¡Pues, también Tú eres! [La traducción es nuestra].

(Я есмь - конечно есь и Tbl!) (Grot, I864: I99).

(Ya yesm'-kaneshna yes'y ty).

Desde luego, muchos antecesores de Derzhavin escribían versos dedicados a este tema. ${ }^{6}$ Sin embargo, el poeta inglés y traductor de la poesía rusa John Bowring (I82I: XIII) afirma: «His ode Bog, Ode on God, is one of the most impressive and sublime addresses I am acquainted with, on a subject so pre-eminently impressive and sublime».7 Por todo ello la obra de Derzhavin ocupa un lugar distinguido entre las composiciones que se han escrito sobre este tema tanto por poetas rusos, como por poetas extranjeros, y tiene un gran éxito no solo en Rusia, sino en todo el mundo. ${ }^{8}$

Es preciso destacar que la Oda al Ser Supremo fue la primera obra de la literatura rusa traducida al español y publicada en las revistas españolas. Este hecho es indudable y se menciona en todos los artículos acerca de las traducciones de la literatura rusa en el mundo hispano. E1 objetivo de este estudio es investigar los detalles de la primera traducción española de la oda de Derzhavin. En la presente nota se trata de establecer la fecha y el nombre del autor de la primera traducción publicada de esta obra

6 Lo más frecuente es que los críticos literarios comparen la oda Dios con el poema religioso El Mesías (I84I) de Friedrich Klopstock, el que Derzhavin leyó en el original y trató de traducir al ruso.

7 «Su Oda Bog, La Oda al Ser Supremo, es una de las alocuciones más impresionantes y sublimes que yo conocía sobre esta cuestión tan eminentemente impresionante y sublime» [la traducción es nuestra].

8 Los interesados pueden leer el volumen editado por Grot (I864) que constituye una buena introducción al tema de la fama internacional de la oda Dios. Entre los trabajos más recientes sobre las traducciones de la oda de Derzhavin a otros idiomas ver, por ejemplo, los artículos de Diomin (2003) y Frolov (2003). poética en español, ya que hasta ahora se había considerado que era una traducción de un autor anónimo. Asimismo, se determina la revista en que la traducción apareció por primera vez y el 105 texto que le sirvió de fuente.

\section{ANÁLISIS BREVE DE LOS ESTUDIOS ANTERIORES}

En primer lugar habría que plantearse la cuestión de quién fue en realidad el autor de la primera traducción de la Oda al Ser Supremo de Derzhavin en español. En los trabajos sobre la historia de la difusión de la literatura rusa en España se suele dar por ciertas las dos siguientes afirmaciones: que el primer artículo sobre la literatura rusa fue escrito por un autor desconocido y fue publicado en I852 en Madrid en la revista literaria ilustrada El Semanario Pintoresco Español; 9 y que la primera traducción española de la Oda al Ser Supremo de Derzhavin, también de un autor desconocido, fue publicada por primera vez en Barcelona en I838 y se hizo desde una traducción francesa ${ }^{\mathrm{Io}}$. La última afirmación

9 Anónimo (1852). Estado actual de la literatura rusa. Semanario pintoresco español, I7, 398-399.

$\mathrm{El}$ artículo se cita como la primera referencia a un estudio dedicado a la exposición de la literatura rusa en España en muchos trabajos, por ejemplo: Borja Rodríguez (2013: 296); Morillas (20II: I20); Monforto (2010: 309); MychkoMegrin, (2007: 49), entre otros.

Io La Bibliografía Russian Literature in the Hispanic World editada por George Schanzer (1972), aborda la problemática de la traducción de la literatura rusa y constituye una buena introducción al tema. Entre los artículos posteriores del 1972 donde se repita el hecho de la primera publicación de la oda de Derzhavin en 1838 hay que mencionar los trabajos más importantes: Bagno (I983). Sobre el tema "Pushkin en España», p. I67; Monforto (2010). «Las ediciones periódicas como un factor clave en la difusión de la literatura rusa durante la segunda mitad del siglo XIX.» Literatura rusa en la prensa hispánica. Traducción y cultural Translation and Culture. Bern, 2010, pp. 308-319; Morillas (20II). «Dostoievski en España». Mundo Eslavo, Io, p.I2o; Morillas (20I2). «La literatura rusa actual en España. Situa- 
no está apoyada por ningún análisis comparativo de los dos textos traducidos y se basa simplemente en la tradición y la práctica que existía en 106
España entre los traductores de los siglos XVIIIXIX (y hasta mediados de siglo xx) que consistía en usar como fuente una traducción francesa y no el texto original en ruso.

En aquella época eran pocos los traductores españoles que supieran ruso y valoraran la literatura rusa. Los contactos entre Rusia y España fueron, en el campo de las letras, indirectos. La razón principal fue que en el siglo xix había poco contacto e intercambio de personas entre los dos países. Juan Valera, que formaba parte de la misión diplomática española en San Petersburgo desde diciembre 1856 hasta junio I857, fue el autor de Las cartas desde Rusia, una obra famosa que permitió a los lectores españoles obtener unos primeros conocimientos de la historia de Rusia, sus costumbres y su literatura. En una de sus primeras cartas de I de enero de I857 Valera hace la siguiente observación acerca del grado de conocimiento de la cultura y la literatura rusa por parte del público español: «La literatura de esta nación (de Rusia-NK) apenas es conocida en parte alguna, y la lengua, aunque empieza a estudiarse, se sabe poco» (Valera, 2005: 83).

Debido a esta barrera lingüística los traductores españoles se vieron obligados a hacer sus traducciones de la literatura rusa a partir de versiones en otros idiomas, por lo general, en francés. De este modo, el lector español conoció las obras de Pushkin, Gogol, Tolstoi y Dostoievski. Ciertamente, las traducciones indirectas en su mayor parte desde el francés, en el papel de mediador, eran un fenómeno bastante común

ción en el mercado español hasta el año 20II». Mundo Eslavo, II, pp. 83-88. Morillas (20I2). «La literatura rusa actual en España. Situación en el mercado español hasta el año 20II». Mundo Eslavo, II, pp. 83-88. en aquella época. No solo era cuestión de proximidad territorial, cultural y lingüística de los dos países, sino también de tradición y prestigio. En los siglos xviı y xıx París, «la ciudad dotada del prestigio literario más grande del mundo», se convirtió en la capital cultural de Europa, «en la capital del universo literario», escribe Pascale Casanova (200I: 40, 4I). Y añade, hablando del triunfo del francés, que «el uso del francés se extiende por toda Europa» y que «la traducción al francés, debido al poder único de consagración de Paris, ocupa un lugar especial» (2001: 96, I96).

En el caso de Derzhavin la primera traducción de su Oda al Ser Supremo al francés fue realizada ya en 1799 por dos jóvenes poetas rusos, Zhukovski y Rodzianko, que regalaron el resultado de su trabajo al autor mismo. Es posible que este hecho inspirara a literatos franceses a realizar sus propias versiones de traducción de esa obra. En total, antes de I855 se publicaron por lo menos I5 traducciones francesas de la Oda al Ser Supremo. Por tanto, no es de extrañar que en los artículos dedicados a la difusión de la literatura rusa en España se afirmara que la primera traducción de la Oda al Ser Supremo de Derzhavin al español se basaba en la traducción francesa. Incluso algunos de los investigadores llegan a la conclusión de que «los defectos de la versión castellana bien pueden ser defectos de una francesa, en prosa, que serviría de base» (Schanzer, I968: 821).

En 1972 George Shanzer publicó en Toronto la primera bibliografía detallada sobre las relaciones literarias hispano-rusas: Russian Literature in the Hispanic World. En el resumen de este trabajo se indica que la cronología de traducciones de la literatura rusa al español comienza con una adaptación del poema de G.R. Derzhavin «Oda al Ser Supremo», de I838 (López-Morillas, I975: 42I). La bibliografía 
de Shanzer incluye 3700 títulos de libros que abarcan colecciones y antologías de literatura rusa, crítica y traducciones individuales en el período desde I838, cuando se publicó la Oda al Ser Supremo, hasta 1965.

Entre los trabajos más recientes hay que mencionar el artículo «Literatura rusa en la prensa hispánica» de Roberto Monforto, donde se analiza el papel de los medios de comunicación en la difusión de la literatura rusa en España en la segunda mitad del siglo xIx. En esta publicación se repite una vez más que la primera obra rusa traducida al español era la Oda al Ser Supremo, que fue publicada en 1838 en la revista La Religión en Barcelona. Luego el autor afirma: «La traducción, como era de esperar, heredó todos los defectos de la traducción francesa de la que, con toda seguridad, deriva» (Monforto, 2010: 308).

A continuación presentamos un breve resumen de las publicaciones sobre el tema.

Habitualmente se hace referencia a las siguientes ediciones como primeras traducciones de la Oda al Ser Supremo de Derzhavin al español:

I. En I838 en la revista La Religión de Barcelona. ${ }^{\text {II }}$

2. En i844 en La Revista Católica de Santiago de Chile. ${ }^{\text {I2 }}$

3. En I884 en la Revista Contemporánea de Madrid. ${ }^{13}$

George Shanzer analiza las tres traducciones y comenta que la Revista Contemporánea de Madrid «indica el traductor, Víctor Suáres Capalleja, y el modelo francés de Eichhoff, cuya fecha es posterior al texto castellano de 1838 . Las versiones barcelonesa y santiagueña son

\footnotetext{
II La Religión: periódico filosófico, histórico y literario (1838), IV, pp. I82-I86.

${ }_{12}$ La Revista Católica (1844), 20, pp. 162-63.

13 Revista Contemporánea (1884), 50, pp. 328-32.
}

idénticas. «La Revista Católica» cita su fuente, «La Religión»; ésta no revela la suya» (Shanzer, 1968: 820).

\section{LA PRIMERA PUBLICACIÓN DE LA ODA AL SER SUPREMO EN LA REVISTA EL CENSOR EN I82I}

De esta forma, tal como acabamos de ver, en todas las publicaciones citadas se considera que el año de la primera traducción de la Oda al Ser Supremo de Derzhavin es 1838. Sin embargo, hemos encontrado que en I82I en la revista política y literaria El Censor de Madrid se publicó aparentemente el primer artículo dedicado a la literatura rusa que incluía una traducción de la oda de Derzhavin en español (Specimens, I821: 422-427). La publicación en El Censor empieza con el título en inglés «Specimens of the Russian Poets: translated by John Bowring. London I82I», seguido de su traducción al español, «Las piezas escogidas de los poetas rusos, traducidas al inglés por el señor Juan Bowring. Londres I82I» y continúa hablando sobre la impresión que la obra había producido entre los lectores: «Las piezas...han obtenido de los literatos británicos un acogimiento superior a las esperanzas del traductor» (Specimens, I821: 40I).

El autor John Bowring era un diplomático, poeta, periodista, editor y traductor políglota. Según la enciclopedia Dictionary of English and American authors, sabía 200 idiomas y hablaba unos roo de ellos (Cousin, I9ro: 44), en particular, llegó a dominar varias lenguas eslavas. ${ }^{\mathrm{I}}{ }^{4}$ Se interesaba por la cultura y la literatura de los países de Europa del Este y escribió antologías poéticas de Polonia (1827), Serbia (I827) y Che-

I4 Sobre la Antología Rusa de John Bowring, la primera traducción de la poesía rusa al inglés, los interesados pueden leer los artículos de Torralbo Caballero (20II), Tsvetkova y Volgina (2009). 
quia (1832). Pero la primera en esta serie de sus trabajos fue la antología de Rusia [Rossiyskaya Antologia] con el título Russian Anthology. 108 Specimens of the Russian Poets publicada en I82I. La obra de John Bowring consta de tres partes: una introducción histórico-cultural detallada y muy extensa, que ocupa 29 páginas del total de 203 páginas del libro, una parte poética, y una tercera parte con unos datos biográficos de los autores. La obra Russian Anthology (Antologia Rusa) de John Bowring incluye unos versos seleccionados de los 13 mejores poetas rusos del siglo XviII y del comienzo del siglo xix. Entre ellos Derzhavin se nombra como el más brillante, y por esa razón, su «magnífica» Oda al Ser Supremo abre la Antología Rusa (Bowring, I82I: 3-9).

Se puede pensar que no fue por causalidad que se publicara la antología de la poesía rusa en español en El Censor. La revista fundada en la época del Trienio liberal, aunque existió poco tiempo, desde 1820 hasta I822, fue uno de los focos relevantes de la vida social, intelectual y cultural de España en los años 20 del siglo xIx. Uno de los fundadores de El Censor fue el destacado literato sevillano Alberto Lista. Como escribe Eugenio de Ochoa en la introducción dedicada a las Poesías de Alberto Lista (Lista, 1953: 27I), el poeta sevillano asociado con escritor satírico y con el helenista y crítico José Gomes Hermosilla, publicó desde Agosto de I820 hasta Julio de I822 los diez y siete tomos de El Censor, «el periódico más importante y mejor redactado que ha existido en España. «Alberto Lista escribió la mayor parte de los artículos de crítica literaria y teatral en la revista El Censons (El Censor, 1820).

$\mathrm{El}$ título «El Censor» «equivale en la época a lo que hoy en día llamaríamos «el crítico» y era bastante familiar al público español porque a finales del siglo xvin existía el periódico El

Censor que imitaba al famoso periódico inglés The Spectator (El Espectador) que era muy popular entre los lectores españoles» (Uzcanga Meinecke, 2003: 7). Se puede suponer que dando el mismo nombre a la revista, sus fundadores tenían la intención de subrayar la sucesión, más concretamente, la continuación de las tradiciones liberales, la tendencia satírica, la crítica de las costumbres tradicionales y de la moral. Los editores y los autores de la revista eran partidarios apasionados de las ideas de la Ilustración. Además era muy importante la política cosmopolita del antecesor, que prestaba mucha atención a los problemas de la historia, la cultura y las costumbres de distintos países.

Tal vez no fuera una casualidad que precisamente en esta revista apareciese el artículo sobre la historia y la cultura de Rusia, de un país tan poco conocido en España en aquellos tiempos, y que presentó a los lectores españoles la poesía rusa del siglo xviı, en particular, la de Gavrila Derzhavin, el último poeta del clasicismo ruso, representante de la época de la Ilustración. Lo más destacable es que la traducción de la oda de Derzhavin no fuera hecha de una versión francesa, como correspondería a la tradición de las traducciones en España en el siglo XIx, sino de un texto en inglés. ${ }^{\text {I5 }}$

\section{AlbERTO LISTA, PRIMER TRADUCTOR DE LA ODA AL SER SUPREMO}

En aquella época pocos eran los traductores que sabían inglés bien y podían traducir obras literarias inglesas. Uno de los primeros escritores que tradujeron la literatura inglesa en España fue el ilustre fundador de El Censor, el sevillano Alberto Lista.

I5 El Censor (I82I), Madrid, Tomo XII, 72: pp. 422-427 
En el estudio fundamental del famoso hispanista alemán Hans Juretschke (I95I: 48), dedicado a la vida y obra del poeta de Sevilla, se afirma, que «Lista repite en varias ocasiones que conocía el inglés, conocimiento que justifica implícitamente en su reseña del libro de lord Holland y en las traducciones de las poesías rusas de Bowring o de Clarisa de Richardson». En el otro capítulo del libro titulado «E1 mundo liberal» y dedicado a las relaciones entre políticos europeos y liberales españoles, Hans Juretschke (1951: 364) menciona el nombre de John Bowring entre los liberales ingleses como «banquero y escritor políglota», que tradujo poesía rusa al inglés. Luego, dice que Lista tradujo varios fragmentos de la obra de Bowring. De esta manera, deducimos que el nombre del autor de la traducción de la Oda al Ser Supremo del inglés al español fue Alberto Lista.

Por el título de uno de los artículos más recientes sobre las traducciones de Lista del inglés- «Alberto Lista: An anglophile pioneer in Spanish translation»-de Juan de Dios Torralbo Caballero, se constata que el autor lo considera como uno de los primeros maestros de la traducción del inglés al español y lo caracteriza (20Ir: 399) «como miembro de un grupo literario en Sevilla que tornó su mirada hacia la literatura inglesa en una época en la que la mayoría de los escritores españoles estaban centrándose en la musa francesa». Alberto Lista tradujo obras poéticas de Milton, Pope y Richardson. En su artículo, Torralbo Caballero (20Ir: 408) también se refiere, entre otras traducciones del inglés de Alberto Lista, a la traducción de Specimens of the Russian Poets de John Bowring. Y aunque no menciona el nombre de Derzhavin ni el título de su famosa oda sí que cita al final del capítulo los cinco primeros versos de la traducción española de la Oda al Ser Supremo incluida en el artículo de El Censor.
El que en este artículo no se citara el nombre de Derzhavin puede explicarse, al parecer, por el hecho de que la publicación de las obras poéticas de Alberto Lista en la Biblioteca de Autores Españoles de 1953, mencionado por el autor del artículo, tampoco diera el nombre del poeta ruso, cuya oda está citada (Lista, 1953:369-370), igual como en la investigación de la vida y obra de Lista en el libro de Hans Juretschke. Cabe señalar, además, que la dificultad de esclarecer la traducción estudiada estriba en el hecho de que, como se indica en las notas de la Colección de 1953, estos fragmentos de la poesía rusa traducidos al español no se han incluido en ninguna edición de las obras poéticas de Lista (Lista, 1953: 369).

De este modo, se puede concluir que Alberto Lista, autor de la traducción de la Introducción histórica-literaria a la Antología Rusa de John Bowring, fue el primero en presentar la literatura rusa a los lectores españoles y el primero en traducir al español la poesía rusa. Además, se puede constatar que la primera traducción de La Oda al Ser Supremo de Derzhavin fue realizada a partir de la versión inglesa, y no de la francesa, como se pensaba anteriormente.

En su artículo Alberto Lista no sólo traduce la Antología, sino que además comparte la opinión a propósito de las declaraciones de John Bowring referentes a la lengua y literatura rusa, sobre las dificultades de la traducción poética, etc. No obstante, el interés principal para el presente estudio consiste en el hecho de que su traducción de la Oda al Ser Supremo fue realizada a partir de la versión inglesa. Alberto Lista admiraba la traducción poética de John Bowring y pedía perdón modestamente ante los lectores de la revista por la imperfección de la traducción española: «Esta traducción se ha hecho en favor de los que no saben el inglés; mas no la damos como una verdadera traducción poética, porque 
le falta mucho para serlo. Si la hemos puesto en verso, es por no destruir enteramente el entusiasmo que reina en la traducción inglesa» (Specimens, I821: 427).

Al comparar las dos publicaciones de la traducción española de la Oda al Ser Supremo de Derzhavin-en El Censor (I82I) y en La Religión (I838) —es fácil descubrir que son completamente idénticas, a excepción de las poco frecuentes inexactitudes ortográficas, gramaticales y léxicas («odeaste»/«rodearse», «se exhalaron»/«se ecshalaron», «existo»/ «ecsisto», «reynos»/«reinos», «aumentada á miríadas, brillando»/ «aumentada y espléndida, brillando») y de pequeñas divergencias ediciones (por ejemplo, en el texto de I8 38 hay una división en estrofas, ${ }^{16}$ mientras que en el texto de i82I faltaba).

Veamos cómo empiezan las dos traducciones:

¡O tú, eterna unidad, cuya presencia

Llena el espacio, el movimiento rige,

Brilla inmudable sobre el raudo vuelo

Del tiempo asolador! ¡Dios sin segundo

Ser sobre todo ser, único y trino!

(El Censor, 1821: 422)

¡O tú, eterna unidad, cuya presencia

Llena el espacio, el movimiento rige,

Brilla inmudable sobre el raudo vuelo

Del tiempo asolador! ¡Dios sin segundo

Ser sobre todo ser, único y trino!

$$
\text { (La Religión, 1838: 183) }
$$

A pesar de la coincidencia completa de los dos textos analizados, en la revista La Religión, como se ha dicho ya, no hay referencias a la publicación más antigua.

Es preciso decir que no solo son idénticas las traducciones de la oda, sino también la introducción breve a esta y se repite por completo el fragmento del artículo de Alberto Lista de El Censor sobre las traducciones de la oda de Derzhavin a las len-

I6 En la versión original en ruso sí que hay división en estrofas, pero distinta a la división del texto publicado en I838 en La Religión. guas japonesa y china, así como aquella impresión extraordinaria—según testimonio del diplomático y viajero ruso V.M. Golovnin — que esta oda había causado al emperador japonés: «Para dar una idea de la poesía rusa sublime de los rusos, copiaremos la magnífica oda de Derzhavin al ser supremo. ${ }^{17} \mathrm{El}$ viajero Golovnin dice que esta sublime composición se ha traducido en Japón por orden del emperador de aquel país, y se ha puesto bordada de oro en el templo de Jeddo. El mismo honor ha recibido en la China. Se ha traducido al chino y al tártaro, y escrita en una pieza de seda finísima está colgada en el palacio imperial de Pekín» (Specimens, I92r: 4I7; Literatura rusa, I838: I82).

Alberto Lista tradujo la Introducción, que había en el texto de John Bowring, pero sin notas (Specimens, I82I:3). El traductor inglés aclara en la primera nota de su traducción de la oda God (Dios)que la historia sobre las traducciones de la oda a las lenguas china y tártara la había oído de los periodistas, de esta manera advirtiendo al lector la duda acerca de los hechos contados ${ }^{18}$, mientras que en el artículo de Lista y en la introducción breve a la traducción de la poesía de Derzhavin en la revista la Religión falta este comentario.

En el mismo encabezamiento del artículo de 1838 , «Literatura rusa. Traducción de la magnífica oda de Derzhavin al Ser Supremo», se copia el epíteto «magnífica» de A. Lista: «Para dar una idea de la poesía sublime de los rusos, copiaremos la magnífica oda de Derzhavin al Ser supremo» (Specimens, 1821: 4I7).

I7 El título de la oda en esta introducción de Lista se escribe en minúscula y el de la publicación en La Religión en mayúscula: al Ser supremo (La Religión, I838: I82). En otro lugar Lista se utiliza el título «oda á Dios» (El Censor, I82I: 405).

I8 J. Grot, el biógrafo de Derzhavin, en sus comentarios a la obra y vida del poeta desmiente esta historia (Grot, I864: I8). 
Por lo tanto, al comparar las dos publicaciones, en la revista El Censor (I82I) y en la revista La Religión (1838), se puede decir, que la traducción de la Oda al Ser Supremo en La Religión fue tomada de la publicación anterior en El Censor.

\section{CONCLUSIONES}

Al final de la investigación se puede llegar a las conclusiones siguientes:

4. La Oda al Ser Supremo de Derzhavin fue traducida al español por la primera vez por el famoso poeta y traductor Alberto Lista.

5. La oda no se tradujo al español desde una versión francesa anónima, sino a partir de la traducción inglesa del famoso poeta y traductor John Bowring.

6. La primera publicación de la Oda en el español fue publicada no en la revista barcelonesa La Religión en 1838, sino en El Censor de Madrid en I82I.

7. El primer artículo dedicado a la literatura rusa, no apareció en 1852 en la revista $E l$ semanario pintoresco español, sino en I82I en la revista El Censor.

Cabe mencionar que de las seis obras de Gavrila Derzhavin incluidas en la Antología Rusa de Bowring, Alberto Lista ha escogido para su traducción la «magnífica» Oda al Ser Supremo como una muestra imprescindible de la mejor y más solemne poesía rusa.

RECIBIDO EN DICIEMBRE DE 2014 ACEPTADO EN MARZO DE 2015 VERSIÓN FINAL DE SEPTIEMBRE DE 2015

\section{REFERENCIAS BIBLIOGRÁFICAS}

\section{Fuentes primarias}

Bowring, J. (1821). Russian Anthology. Specimens of the Russian Poets: translated by John Bowring. London: Printed by Richard and Arthur Taylor,
Shoe-Lane.

«Literatura rusa. Traducción de la magnífica oda de Derzhavin al Ser Supremo» (1838). La Religión: periódico filosófico, histórico y literario. Barcelona: Imprenta de Brusi. Tomo IV, Pp. I82-I86. "Specimens of the Russian Poets: translated by John Bowring» (1821). El Censor, periódico politico y literario. Madrid, XII/72, pp. $4 \mathrm{OI}^{-} 427$.

\section{Fuentes secundarias}

Borja Rodríguez, G. (2013). «La narrativa en la ilustración (I849-1857): La «serie B» del Semanario Pintoresco Español». Anales, 25, pp. 283-303.

Bregante, J. (2003). "Alberto Lista». Diccionario de literatura española de Jesús Bregante. Madrid: Diccionario Espasa, pp. 493-494.

Casanova, P. (200I). La república mundial de las letras. Trad. de Jaime Zulaika.Barcelona: Anagrama.

Cousin, J. (I9ro). «John Bowring» [en línea]. Dictionary of English and American authors.

London: J. M. Dent \& Sons, LTD. New York: E. P. Button \& co.

https://ia70040o.us.archive.org/28/items/shortbiographicaoocousuoft/shortbiographicaoocousuoft.pdf. [Consulta: Io abril 2015.]

Cueto, L. A. (Ed.) (I953). «Alberto Lista. Poesías». Poetas líricos del siglo XVIII. Biblioteca de Autores Españoles. Madrid: Rivadeneyra.

Diómin, A.O. (2003). «G.R.Derzhavin i itlianski poety (Moretti, Petrarka, Tasso, Metastazio)».

G.R.Derzhavin v novom tysiacheletii. Kazán, Kazanski gosudarstvenyi universitet.

[Diómin, A.O. (2003). «G.R.Derzhavin y los poetos italianos (Moretti, Petrarca, Tasso, Metastasio)». G.R.Derzhavin en el nuevo milenio. Kazán, Universidad Estatal de Kazan].

Frolov, G.A. (2003). «Oda G.R. Derzhavin «Bog» v nemetskom literaturnom kontekste (istochniki, perevody).» G.R.Derzhavin v novom tysiacheletii. Kazán, Kazanski gosudarstvenyi universitet. [Frolov, G.A. (2003). «G.R.Derzhavin en el contexto literario alemán (fuentes y traducciones)». G.R.Derzhavin en el nuevo milenio. Kazán, Universidad Estatal de Kazán].

Juretschke, H. (1952). Vida, obra y pensamiento de Alberto Lista. Madrid: csic.

López-Morillas, J. (I975). «G. O. Schanzer. Russian Literature in the Hispanic World: A Bibliography» (Book Review). Bulletin of Hispanic Studies, 
Liverpool University Press, 52/4, pp. 42I-422.

Monforto, R. (20I0). «Las ediciones periódicas como un factor clave en la difusión de la literatura rusa durante la segunda mitad del siglo XIX ». Traducción y cultura / Translation and Culture: La literatura traducida. Relaciones literarias en el ámbito hispánico: traducción, literatura y cultura. Bern: Editorial científica internacional, pp. 308-319.

Morillas, J. (20II). «F.M. Dostoievski en España». Mundo Eslavo, io, pp. II9-I43.

- (20I2). «La literatura rusa actual en España. Situación en el mercado español hasta el año 2OII». Mundo Eslavo, II, pp. 83-88.

Mychko-Megrin, I. (2007). «Recepción de la literatura rusa soviética en España: E1 Maestro y Margarita y otras obras de Mijaíl Bulgákov». Transfer II: 2 , pp. $49^{-5} 8$.

Schanzer, George O. (I970). «Las primeras traducciones de literatura rusa en España y en América». Actas del Tercer Congreso Internacional de Hispanistas, pp. 815-822.

- (1972). Russian Literature in the Hispanic World (la Literatura Rusa En el Mundo Hispánico): A Bibliography. Toronto: University of Toronto Press.

Tsvetkova, M.V. y Volgina, A.S. (2009). «Ruskaya antologia John Bowring kak pervyi opyt ruskoi poezii v angliiskom perevode». Rossiyskaya $y$ zarubezhnaya filologia. Vestnik

Permskogo universiteta, 3, 52-29 [Tsvetkova, M.V. y Volgina, A.S. (2009). "Antología Rusa de John Bowring como la primera obra poética rusa en la traducción inglesa.»

Filologia rusa y extranjera. El Mensajero de la Universidad de Perm, 3, 52-29].

Torralbo Caballero, J. (2oII). «Alberto Lista: An anglophile pioneer in Spanish translation». Entreculturas, 3, pp. 399-4I3.

Valera, J. (2005). Cartas desde Rusia. Madrid: Miraguano Ediciones.

Uzcanga Meinecke, F. (2003) «El Censor». Clásicos y modernos. Barcelona. Crítica. 